\title{
TRATAMIENTO ENDOSCÓPICO EN REFLUJO VESICOURETERAL (RVU). COMPARACION DE DIFERENTES SUSTANCIAS. RESULTADOS A LARGO PLAZO
}

José Manuel Escala Aguirre, Yair Cadena González, Gabriela Retamal Pinto, Pedro-José López Egaña, Nelly Letelier Cancino y Ricardo Zubieta Acuña.

Servicio de Urología y Servicio de Cirugía Infantil. Hospital Exequiel González Cortes. Santiago. Chile

Resumen.- OBJETIVO: El RVU es una de las patologías más frecuentes en la urología pediátrica, clásicamente se ha manejado con tratamiento médico o quirúrgico dependiendo de la edad, grado u otras variables. En los últimos años se ha comenzado a utilizar el tratamiento endoscópico, con distintas sustancias, el cual siendo quirúrgico es mínimamente invasivo. El objetivo de este trabajo es evaluar nuestros resultados con este método.

MÉTODOS: Entre 1996 y el 2004 se utilizó tratamiento endoscópico en 41 pacientes (70 uréteres) usando diferentes sustancias. Se analizó el grado y lateralidad del RVU, mejoría con 1 o más inyecciones, control post operatorio con clínica, ecografía renal vesical y uretrocistografía, y se compararon los resultados con las distintas sustancias usadas a largo plazo, evaluando principalmente las recidivas y/o cuadros de infección urinaria.

RESULTADOS: 41 pacientes entraron al estudio, se inyecto colágeno en 13, Macroplastique ${ }^{\circledR}$ en 14 y en 14 Deflux ${ }^{\circledR} .29$ pacientes recibieron inyección bilateral, resultando un total de 70 uréteres inyectados Con colágeno hubo 53\% de éxito con la primera inyección y $77 \%$ con la segunda, con Macroplastique $\Theta$ hubo $83 \%$ de éxito con la primera inyección y $91 \%$ con la segunda y con Deflux $\Theta 84 \%$ de éxito con la primera inyección Y 88\% con la segunda inyección. El seguimiento post operatorio fue en promedio de 44 meses, con un rango de 18 a 86 meses. 18\% presentó ITU post operatoria la que sé presento en pacientes con falla en el tratamiento. En el seguimiento no hay episodios de ITU en los pacientes con éxito en la aplicación de la sustancia.

CONCLUSIONES: El tratamiento endoscopio es una herramienta útil en el manejo del RVU a largo plazo, ya sea como tratamiento definitivo o como alternativa al manejo médico convencional, obteniendo mayor respuesta con Macroplastiqueß y Deflux $\circledast$.

Palabras clave: Reflujo vésico ureteral. Endoscópico.

Summary.- OBJECTIVES: The VUR is one of the most freJose Manuel Escala A.

Yair Cadena González

Unidad Urología Pediátrica

Hospital Exequiel González Cortes

Barros Luco 3301, San Miguel

Santiago. (Chile).

imescala@clinicalascondes.cl

yaircadena@yahoo.com quent pathologies in pediatric urology. Classically it has been managed with medical or surgical treatment depending on age, grade, and other variables. Over the last years, urologists have started to perform endoscopic treatment with various substances, surgery but minimally invasive. The objective of this work is to evaluate our results with this method.

METHODS: Between 1996 and 2004 we performed endoscopic treatment on 41 patients (70 ureters) using different subs- 
tances. We analyze VUR grade and side, improvement with one or more injections, post-operative follow-up with clinical evaluation, bladder ultrasound and voiding cystourethrogram (VCUG), and compare the long-term results of the different substances used, mainly with the evaluation of recurrences and/or urinary tract infections.

RESULTS: 41 patients entered the study; collagen was injected in 13 cases, Macroplastique ${ }^{\circledR}$ in 14 , and Deflux ${ }^{\circledR}$ in 14 . Twenty-nine patients underwent bilateral injection, adding up to a total of 70 injected ureters. Collagen injection had a success rate of $53 \%$ with the first injection and $77 \%$ with the second, Macroplastique ${ }^{\circledR}$ success rate was $83 \%$ with the first injection and $91 \%$ with the second, and Deflux $\AA 84 \%$ on first injection and $88 \%$ with the second. Mean postoperative follow-up was 44 months, with a range of 18-86 months. $18 \%$ of the patients presented post operative UTI, they were treatment failures. There are no UTI episodes in patients after successful treatment.

CONCLUSIONS: Endoscopic treatment is a useful tool in the long-term management of VUR, both as definitive treatment or as an alternative to conventional medical management, with better results when using Macroplastique $\Theta$ and Deflux $\Theta$.

Keywords: Vesicoureteral reflux. Endoscopy.

\section{INTRODUCCIÓN}

El Reflujo Vésico Ureteral (RVU) primario es una patología frecuente en la consulta urológica pediátrica, constituyendo la causa frecuente de infección urinaria ( 30 a $50 \%$ ) y es el causante de 3 a $23 \%$ de la insuficiencia renal en niños (1-3).

Debido a esto, es fundamental realizar un diagnóstico oportuno acompañado de un buen tratamiento y seguimiento adecuado. Las opciones de tratamiento están determinadas dependiendo de la edad de niño, del grado del reflujo y del tiempo de seguimiento.

Habitualmente los pacientes que no tienen indicación quirúrgica permanecen con quimioprofilaxis y control durante períodos prolongados, con el fin de mantener la orina estéril y esperar la resolución espontánea del reflujo (4). Buscando disminuir este período de tiempo se ha comenzado a utilizar el tratamiento endoscópico, el cual siendo quirúrgico es mínimamente invasivo.

Desde la década de los 80 por vía endoscópica se han inyectado diversas sustancias $(5,6)$ con el fin de mejorar la competencia de la unión ureterovesical, desde el teflón, el cual fue abandonado por sus características migratorias, pasando por el Colágeno, Macroplastique $\AA$ sustancias autólogas, hasta el Deflux®. Todos con la finalidad de encontrar una sustancia sin efectos adversos, no inmunológica, que no migre ni se reabsorba, y ojalá de inyección fácil. (7).

\section{MATERIAL Y MÉTODO}

Se revisaron todos los pacientes en quienes se realizó tratamiento endoscópico del reflujo vésico ureteral en la Unidad de Urología Infantil del Hospital Exequiel González Cortés entre junio de 1996 hasta septiembre de 2004. Se tomaron los pacientes con indicación quirúrgica, ya sea por falla en el tratamiento médico, infecciones a repetición, lesión renal, o deseo de terminar con el manejo médico.

Los materiales utilizados fueron colágeno, Macroplastique ${ }^{\circledR}$ (elastómeros de silicona suspendidas en gel bioexcretable) y Deflux ${ }^{\circledR}$ (microesferas de dextranomero en solución hialurónica de sodio).

En todos los casos se realizó el procedimiento bajo anestesia general, en posición de litotomía, se usa cistoscopio con canal de trabajo recto y con video en todos los casos. El volumen inyectado fue variable y dependió de lo observado por el cirujano durante el procedimiento. Los procedimientos fueron ambulatorios (Figuras 1 y 2 ).

Se analizaron antecedentes generales, sustancia utilizada, mejoría con 1 o más inyecciones, resultados y seguimiento. El control post operatorio se realizó con clínica, ecografía renal-vesical a la semana y uretrocistografía 3 meses postoperatorios. Curación se determinó como ausencia de reflujo en la cistografía de control y la no aparición de ITU en el periodo de seguimiento.

Se compararon los resultados de las sustancias entre sí, bajo prueba de Fisher' Irving. Con un error alfa < de $5 \%$ y un poder de $80 \%$.

\section{RESULTADOS}

Un total de 41 pacientes entraron al estudio. A 13 pacientes se les inyectó colágeno (8 bilaterales), 14 fueron inyectados con Macroplastique $\AA$ (10 bilaterales) y en 14 Deflux $\AA$ ( 11 bilaterales), siendo en total 70 uréteres inyectados.

Un $80 \%$ de los analizados son mujeres, la mayoría tiene entre 3 y 8 años $(75 \%)$, con un rango de 1 a 14 años y una media de 6 años. El tiempo de seguimiento promedio fue de 44 meses.

Se observó que de los 70 uréteres estudiados $21 \%$ tenía RVU grado I, $23 \%$ grado II, $36 \%$ grado III, $19 \%$ grado IV y un $1 \%$ tenía RVU grado V.

Se dividieron los datos según el tipo de sustancia inyectada. Los tres grupos no presentaban diferencias significativas prequirurgicamente. En 21 unidades ureterales se inyectó colágeno (13 pacientes), se observó curación del RVU con 1 inyección en 11 uréteres $(53 \%)$ y con 2 inyecciones mejoraron 5 uréteres más, sumando en total 16 uréteres curados $(77 \%)$.

Con Macroplastique $\AA$ se inyectaron 24 uréteres (14 pacientes), mejorando con una inyección 20 uréteres 


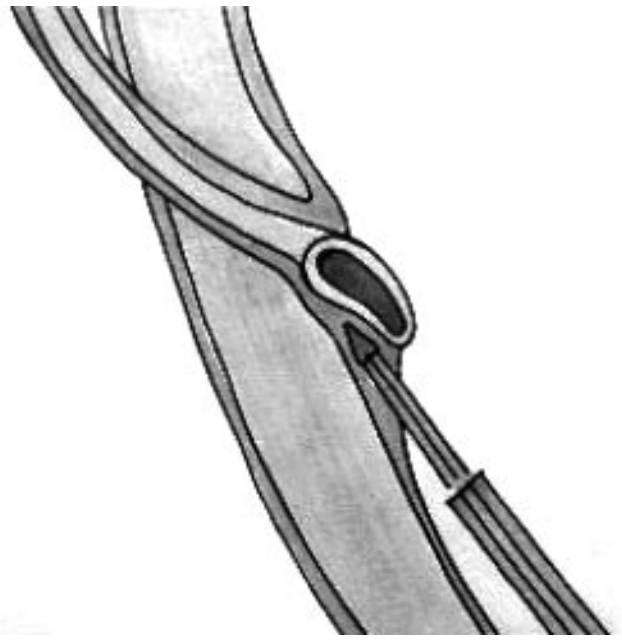

FIGURA 1. Como se inyecta de manera subureteral la sustancia a utilizar para aumentar el trayecto submucoso del uréter.

$(83 \%)$ y con la segunda 2 uréteres más, sumando 22 uréteres $(91 \%)$.

Con Deflux ${ }^{\circledR}$ se inyectaron 25 uréteres (14 pacientes) con éxito en 21 uréteres a la primera inyección (84\%), a 1 paciente portadora de RVU unilateral debió realizársele un neoimplante tipo Cohen debido a la presencia de doble sistema y recidiva del reflujo en sistema inferior y aparición en el sistema superior, los otros 3 uréteres recibieron una segunda inyección con éxito en uno de los tres dando una efectividad total del $88 \%$

Entre las diferentes sustancias se vio que el Macroplastique $₫$ y el Deflux $®$ presentaron mayor efectividad comparándolos con el grupo de pacientes a los que se les aplicó colágeno ( $p>0.05)$. No se presentaron diferencias significativas en la efectividad del Deflux $₫$ y el Macroplas-

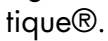

Complicaciones post operatorias, se presentaron

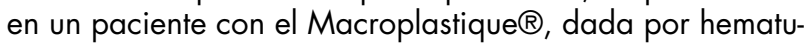
ria e hidroureteronefrosis importante secundaria a solidificación del material intraureteral, que debió ser extraído al séptimo día post operatorio por cistoscopia. No se presentaron otras complicaciones. No se encontró relación con el volumen inyectado ni con la lateralidad. En los pacientes en que se resolvió el reflujo no se presentaron episodios de ITU en el tiempo de seguimiento.

\section{DISCUSIÓN}

El RVU es una patología potencialmente grave (2). El manejo endoscópico a tomado fuerza debido a la facilidad del mismo, por lo que se han desarrollado diversas sustancias para su uso (8-10). A pesar de que aún no se ha encontrado la sustancia ideal para la inyección subureteral endoscópica en el tratamiento del reflujo tanto el Macroplastique $\AA^{\circledR}$ como el Deflux $®$ han mostrado diferencias significativas en cuanto a sus resultados al compararlas con

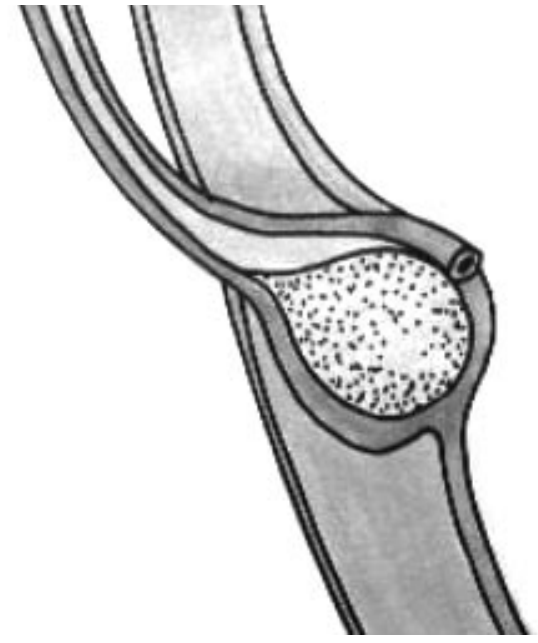

FIGURA 2 Como queda post inyección.

las sustancias más antiguas. Además que se han mostrado como sustancias mas seguras ya que no migran y no son antigénicas, acercándose a la sustancia ideal $(11,12)$.

En este estudio se tomaron los pacientes a los cuales se les realizó manejo endoscópico del reflujo vésico ureteral con diversas sustancias. Se muestra en el mismo que los mejores resultados se dan con el Macroplastique ${ }^{\circledR}$ y Deflux ${ }^{\circledR}$, no así con el colágeno. Datos que se han visto son concordantes con lo escrito en la literatura $(12,13)$. Así mismo al realizar una segunda inyección se alcanza mayor eficacia en el procedimiento.

Complicaciones no se presentaron en los pacientes que recibieron colágeno y Deflux $\circledast$, no se presentó hematuria y en todos los casos el procedimiento fue ambulatorio. No se encontró hidronefrosis secundaria al uso de los agentes inyectables, que está descrita en escasos casos (14). Se presentó en un caso una complicación importante dado por la solidificación del material en la luz ureteral con el Macroplastique ${ }$, tal vez originado por una aplicación cercana a la luz ureteral o incluso dentro de la misma, complicación que no encontramos descrita en la literatura.

Dentro de los agentes usados, nosotros preferimos el uso de Deflux® por la facilidad técnica de su inyección: menor viscosidad, y no requiere de instrumental especial (pistola), no se presentaron complicaciones con el mismo y tiene una eficacia demostrada en diversos estudios (1517). Algunos trabajos muestran además la duración en el tiempo de estas sustancias (18).

A la luz de resultados exitosos recientes, en muchos centros se está utilizando el tratamiento endoscópico para el RVU como tratamiento de primera línea, incluso reemplazando al tratamiento médico, que es largo, tedioso y mal cumplido para muchos pacientes y sus padres, demostrándose una adherencia a la profilaxis incluso menor al $20 \%$ en algunos estudios. Además aprovecha de evitar la realización de frecuentes exámenes, y de exposición a 
múltiples radiaciones. Por otra parte los costos, si bien es cierto no son menores, al comparar con tratamientos prolongados o cirugías que requieren mayor estadía hospitalaria, también se ven disminuidos, aunque sea necesaria una segunda inyección (16). En los pacientes se logró un seguimiento en promedio de 30 meses sin que se presentaran episodios de ITU en el tiempo estudiado, lo que nos muestra le efectividad de las sustancias en el tiempo, permitiendo a los pacientes la suspensión más rápida de la profilaxis, en los pacientes en los que se logró éxito con la inyección, como se muestra también en otros estudios (8).

Es evidente el menor trauma que ocasiona el tratamiento endoscópico en nuestros pequeños pacientes, por lo que asociado a un buen resultado en la era de lo minimamente invasivo, éste debiera convertirse en la primera elección del tratamiento del reflujo vésicoureteral pediátrico en pacientes seleccionados.

\section{CONCLUSIONES}

El manejo endoscópico del reflujo vésico ureteral es rápido, sencillo y eficaz. Diversas sustancias están al alcance para la realización de este tipo de procedimientos, siendo en este estudio mas efectivo el uso del Deflux $\otimes^{\circledR}$ y del

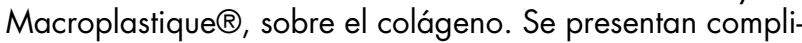
caciones escasas con todos los materiales. Sin embargo, dada la facilidad de aplicación, recomendamos el uso del Deflux $®$ para el manejo endoscópico del reflujo vésico ureteral en pacientes pediátricos seleccionados y con buenos resultados a largo plazo.

\section{AGRADECIMIENTOS}

A la Corporación Renal Infantil M.A.T.E.R. por su apoyo en los trabajos de investigación.

\section{BIBLIOGRAFÍA y LECTURAS RECOMENDADAS ("lectura de interés $\mathrm{y}^{* *}$ lectura fundamental)}

1. FREY, P.; LUTZ, N.; JENNY, P. y cols.: "Endoscopic subureteral injection for the treatment of vesicoureteral reflux in infants and children”. J. Urol., 154: 804, 1995.

2. CONNOLLY, L.P.; ZURAKOWSKI, D.; CONNOLLY, S.A. y cols.: "Natural history of vesicoureteral reflux in girls after age 5 years". J. Urol., 166: 2359, 2001.

3. CHERTIN, B.; PURI, P.: "Familial vesicoureteral reflux". J. Urol., 169: 1804, 2003.

*4. MEDICAL VERSUS SURGICAL TREATMENT OF PRIMARY VESICOURETERAL REFLUX: "Report of the International reflux study committee". Pediatrics, $67: 392,1981$.
5. MATOUSCHEK, E.: "The management of vesicoureteral reflux using transurethral injections of Teflon paste [in German]". Urologe A., 20: 263, 1981.

6. O'DONNELL, B.; PURI, P.: "Treatment of vesicoureteric reflux by endoscopic injection of Teflon". Br. Med. J., 289: 7, 1984.

**7. KEATING, M.A.: "Role of periureteral injections in children with vesicoureteral reflux". Curr. Opin. Urol., 15: 369, 2005.

*8. CALDAMONE, A.; DIAMOND, D.: "Long term results of the endoscopic correction of vesicoureteral reflux in children using autologous chondrocytes". J. of Urol., 165: 2224, 2001.

9. CHERTIN, B.; PURI, P.: "Endoscopic Manegement of Vesicoureteral Reflux: Does It Stand the Testo of Time?". European Urology, 42: 598, 2002.

10. VAN CAPELLE, J.W.; DE HAAN, T.; EL SAYED, W. y cols.: "The long-term outcome of the endoscopic subureteric implantation of polydimethylsiloxane for treating vesico-ureteric reflux in children: A retrospective analysis of the first 195 consecutive patients in two european centres". BJU Int., 94: 1348, 2004.

11. STEYAERT, H.; SATTONNET, C.; BLOCH, C. y cols.: "Migration of PTFE paste particles to the kidney after treatment for vésico-ureteral reflux". BJU Int., 85: $168,2000$.

*12. CAPOZZA, N.; LAIS, A.; NAPPO, S. y cols.: "The Role of Endoscopic Treatment of Vesicoureteral Reflux: a 17-year experience". The Journal of Urology, 172: 1626, 2004.

13. OSWALD, J.; RICCABONA, M.; LUSUARDI, L. y cols.: "Prospective comparison and 1 year follow up of a single endoscopic subureteral polydimethylsiloxane versus Dextranoner/hyaluronic acid copolimer injection for treatment of vesicoureteral reflux in children". Urology, 60: 894, 2002.

*14. VANDERSTEEN, D.R.; ROUTH, J.C.; KIRSCH, A.J. y cols.: "Postoperative Ureteral Obstruction After Subureteral Injection of Dextranomer/Hyaluronic Acid Copolymer". The Journal of Urology, 176: 1593, 2006.

15. KIRSCH, A.; PÉREZ-BRAYFIELD, M.; SCHERZ, H.: "Minimamally invasive treatment of vesicoureteral reflux with endoscopic injection of dextranomer/hyaluronic acid copolymer: The children`s hospital of Atlanta experience”. J. Urol., 170: 211, 2003.

16. PURI, P.; CHERTIN, B.; VELAYUDHAM, M. y cols.: "Treatment of vesicoureteral reflux by endoscopic injection of dextranomer/hyaluronic acid copolymer: Preliminary resuls". J. Urol., 170: 1541, 2003.

**17. ELDER, J.S.; DIAZ, M.; CALDAMONE, A.A. y cols.: "Endoscopic Therapy for Vesicoureteral Reflux: A Meta-Analysis. I. Reflux Resolution and Urinary Tract Infection". The Journal of Urology, 175: 716, 2006.

**18. LACKGREN, G.; WAHLIN, E.; SKOLDENBERG, E. y cols.: "Long term follow up of children treated with dextranomer/ Hyaluronic acid copolymer for vesicoureteral reflux". The Journal of Urology, 166: 1887, 2001. 